\title{
A BERNSTEIN-GABBER-JOSEPH THEOREM FOR AFFINE ALGEBRAS*
}

\author{
by V. V. BAVULA and T. H. LENAGAN
}

(Received 12th May 1997)

\begin{abstract}
Bernstein's famous result, that any non-zero module $M$ over the $n$-th Weyl algebra $A_{n}$ satisfies $\mathrm{GKdim}(M) \geq G \operatorname{Gdim}\left(A_{n}\right) / 2$, does not carry over to arbitrary simple affine algebras, as is shown by an example of McConnell. Bavula introduced the notion of filter dimension of simple algebra to explain this failure. Here, we introduce the faithful dimension of a module, a variant of the filter dimension, to investigate this phenomenon further and to study a revised definition of holonomic modules. We compute the faithful dimension for certain modules over a variant of the McConnell example to illustrate the utility of this new dimension.
\end{abstract}

1991 Mathematics subject classification: 16P90, 16P40, 16D60, 16 S32.

\section{Introduction}

In [4], Bernstein proved his famous result that any nonzero module over the Weyl Algebra $A_{n}(\mathbb{C})$ has Gelfand-Kirillov dimension at least $n$. The finitely generated $A_{n}(\mathbb{C})-$ modules $M$ for which $\mathrm{GKdim}(M)=n$ are called holonomic modules. Bernstein used the fact that holonomic $A_{n}(\mathbb{C})$-modules have finite length to give a beautiful proof of a result, conjectured by I. M. Gelfand, on the analytic continuity of certain functions defined on the half plane $\mathfrak{R}\{z\}>0$. A discussion of these results is given in Chapter 8 of [5].

Since $\operatorname{GKdim}\left(A_{n}(\mathbb{C})\right)=2 n$, Bernstein's result can be rewritten in the form $\operatorname{GKdim}\left(A_{n}(\mathbb{C})\right) / 2 \leq \mathrm{GKdim}(M)$, for all nonzero $A_{n}(\mathbb{C})$-modules $M$.

Gabber, generalising a result of Joseph, extended Bernstein's result to show that if $\mathfrak{g}$ is any finite dimensional algebraic Lie algebra over an algebraically closed field $K$ of characteristic zero and $M$ is a finitely generated left $U(\mathfrak{g})$-module, where $U(\mathfrak{g})$ is the universal enveloping algebra of $\mathfrak{g}$, then $G K \operatorname{dim}(U(\mathfrak{g}) / \operatorname{ann}(M)) / 2 \leq \mathrm{GKdim}(M)$, see [5, Chapter 9] or [6, Proposition 6.1.4].

In contrast, there is an example, due to McConnell, [7], of a simple affine algebra such that $\mathrm{GKdim}(A)=n$ but $A$ has a simple module of Gelfand-Kirillov dimension one. This algebra is a homomorphic image of the enveloping algebra of a finite dimensional solvable Lie algebra; so the algebraic condition in Gabber's Theorem cannot be removed.

- This research was done while the first author was visiting the Department of Mathematics at the University of Edinburgh, supported by a grant from the Centenary Fund of the Edinburgh Mathematical Society. 
In order to reconcile these results, the first author has recently introduced the notion of the filter dimension of a module, $\mathrm{fd}(M)$, and has shown that if $A$ is any simple affine algebra then

$$
\frac{\mathrm{GKdim}(A)}{\mathrm{fd}(A)+\max \{\mathrm{fd}(A), 1\}} \leq \mathrm{GKdim}(M)
$$

for any nonzero finitely generated $A$-module $M,[1]$.

The Krull dimension of a module, in the sense of Gabriel and Rentschler, is one of the most useful invariants of a module, but it is notoriously difficult to calculate its exact value in general. Whenever the Gelfand-Kirillov dimension is well-behaved and one can obtain a lower bound on the Gelfand-Kirillov dimension of nonzero modules then there is a hope that one can establish upper bounds on Krull dimension. For example, in [5, Corollary 7.12], it is shown that if $A$ is any almost commutative algebra then $\mathrm{GKdim}(A) \geq \mathrm{Kdim}(A)+s(A)$, where $s(A)$ is the minimal possible dimension for nonzero $A$-modules. This idea was used by S. P. Smith, [10], to show that the Krull dimension of the enveloping algebra of $\operatorname{sl}(2, \mathbb{C})$ is two, rather than the previously believed value of three.

In [2], the first author has used the above inequality to establish that

$$
\mathrm{K} \operatorname{dim}(A) \leq \mathrm{GKdim}(A)\left(1-\frac{1}{\mathrm{fd}(A)+\max \{\mathrm{fd}(A), 1\}}\right)
$$

for any simple affine left finitely partitive algebra $A$ with $\operatorname{GKdim}(A)<\infty$.

In this paper we address the question of whether there is any reasonable version of these results in general affine algebras.

If we are going to make a comparison between $\operatorname{GKdim}(M)$ and $\operatorname{GKdim}(A)$ then the first thing to do is to factor out the annihilator of $M$. Also, if we are interested in lower bounds for Gelfand-Kirillov dimension then we will be considering simple modules. Thus, any analysis of the general situation will have to consider the primitive factors of the algebra. Taking account of these requirements, we introduce a generalisation of the filter dimension called the left faithful dimension. A lower bound $S_{A}$ on possible Gelfand-Kirillov dimensions of modules is established, by using the left faithful dimension. This leads to the standard comparison between Krull and Gelfand-Kirillov dimension for reasonably behaved rings.

Of course, if there are any finite dimensional simple modules over the algebra $A$ then these results reveal nothing, since in this case $S_{A}=0$. However, if this is the case and there is a finite dimensional module $M$ then $A / \operatorname{ann}(M)$ is a finite dimensional algebra, and the study of the modules can safely be left to our finite dimensional colleagues! Thus, in order to obtain any meaningful interpretation of our results, we will need to discuss algebras with no finite dimensional images. In later work we hope to return to the problem of dealing with the non-finite dimensional modules over algebras which do have some finite dimensional images.

The classical definition of a holonomic module arises from the work of Bernstein 
and Gabber: a module $M$ is holonomic if $\operatorname{GKdim}(A / \operatorname{ann}(M)) / 2=\mathrm{GKdim}(M)$. In the Bernstein-Gabber setting these modules have least possible Gelfand-Kirillov dimension and are very well behaved; for example, a finitely generated holonomic module has finite length. In view of McConnell's example, it is clear that to extend the definition of holonomic to a wider class of algebras' we should consider modules with least possible Gelfand-Kirillov dimension $S_{A}$. This presents difficulties in. that $S_{A}$ is an infimum, and so it is not clear at the outset that there will be modules which achieve this dimension. By imposing certain reasonable finiteness conditions, we show that holonomic modules in the new sense exist and that finitely generated holonomic modules have finite length.

Of course, simple non-finite dimensional algebras fall within our discussions; so it is appropriate to consider the relationship between filter dimension and left faithful dimension in this setting, and to check that our new notion of holonomicity coincides with the classical definition. One class of algebras where this can be done is the class of rings of differential operators in the case that the base ring is a commutative regular integral domain. The first author has considered the behaviour of filter dimension in this setting [2] and established that the filter dimension is one. We show that the left faithful dimension is also one for holonomic modules and that in this setting the classical and new definitions of holonomic module coincide.

One of the difficulties of any of these approaches is that of calculating dimensions in interesting classes of rings, and even establishing the existence of holonomic modules. In the penultimate section we consider a class of algebras called Schurian Algebras. These are $K$-algebras $A$ such that each simple module $M$ has $\operatorname{End}_{A}(M)=K$. For a faithful simple module $M$ over such an algebra, the algebra acts as a dense ring of $K$-linear transformations on $M$ and we introduce a new growth function, the Schur dimension, which measures the rate at which the integer $j$ grows so that $\operatorname{Hom}\left(M_{i}, M_{i}\right) \subseteq A_{j}$, for filtrations $\left\{A_{i}\right\}$ of $A$ and $\left\{M_{i}\right\}$ of $M$. We establish a relationship between the Schur dimension and the left faithful dimension which we hope will prove useful in calculating dimensions. In particular, in the differential operator case mentioned above, we establish that the Schur dimension of a simple module is greater than or equal to one and if the Schur dimension is one then the module is holonomic. However, Stafford's examples of nonholonomic modules over Weyl algebras provide examples of modules with Schur dimension greater than one.

In the final section, we consider a multiplicative analogue $A=A(n)$ of the McConnell example referred to above, and show that, as with the McConnell example, $A$ has simple modules $M_{1}, M_{n}$, of Gelfand-Kirillov dimensions 1 and $n$, respectively. We also calculate the left faithful dimension for both modules: we show that the left faithful dimension of $M_{1}$ is $n$, and that the left faithful dimension of $M_{n}$ is $\frac{1}{n}$.

\section{Left faithful dimension}

Let $K$ be a field and let $A:=K\left(x_{1}, \ldots, x_{s}\right)$ be an affine $K$-algebra. The algebra $A$ is equipped with a standard finite dimensional filtration $F: A=\cup_{i \geq 0} A_{i}$, where 


$$
A_{0}=K \subseteq A_{1}=K+\sum_{i=1}^{s} K x_{i} \subseteq \ldots \subseteq A_{i}:=A_{1}^{i} \subseteq \ldots
$$

Let $M$ be a finitely generated $A$-module with a finite dimensional subspace $M_{0}$ such that $M=A M_{0}$. There is a standard finite dimensional filtration, $\left\{M_{i}\right\}$, of $M$ given by $M_{i}:=A_{i} M_{0}$.

Assume that $M$ is a faithful $A$-module. For any nonzero element $a \in A$, there exists a least integer $i$ such that $a M_{i} \neq 0$. Set $n_{M, F, M_{0}}(a)$ to be this least integer $i$. For convenience, set $n_{M, F, M_{0}}(0):=0$.

For any subset $V$ of $A$, set

$$
n_{M, F, M_{0}}(V):=\sup \left\{n_{M, F, M_{0}}(v) \mid v \in V\right\}
$$

Lemma 1. Let $A$ be an affine algebra and let $M$ be a finitely generated faithful $A$ module. Then, $n_{M, F, M_{0}}(V)<\infty$ for any finite dimensional subspace $V$ of $A$.

Proof. Suppose that the result is false, so that $n_{M, F, M_{0}}(V)=\infty$ for some finite dimensional space $V$ of $A$. Choose a sequence $a_{1}, a_{2}, \ldots$ of elements of $A$ such that $n\left(a_{1}\right)<n\left(a_{2}\right)<\ldots$. For each $i \geq 1$, set $V_{i}=\sum_{i \leq j} K a_{j}$. Thus, there is a descending sequence of subspaces

$$
V \supseteq V_{1} \supseteq V_{2} \supseteq \ldots
$$

Since $V$ is finite dimensional, this descending sequence must terminate, say at $V_{m}=V_{m+1} \ldots$. Since $a_{m} \in V_{m}=V_{m+1}$, there are scalars $\alpha_{i}$, for $i>m$ and with only finitely many $\alpha_{i} \neq 0$, such that $a_{m}=\sum_{i>m} \alpha_{i} a_{i}$.

Hence,

$$
0 \neq a_{m} M_{n\left(a_{m+1}\right)-1} \subseteq \sum_{i>m} \alpha_{i} a_{i} M_{n\left(a_{m+1}\right)-1} \subseteq \sum_{i>m} \alpha_{i} a_{i} M_{n\left(a_{i}\right)-1}=0
$$

a contradiction.

Since each of the subspaces $A_{i}$ of the standard filtration of $A$ is finite dimensional, the following definition makes sense.

\section{Definition 2.}

$$
n(i):=n_{M, F, M_{0}}\left(A_{i}\right), \quad i \geq 0 .
$$

In other words, $n(i)$ is the least integer $j$ such that the $K$-linear map

$$
A_{i} \rightarrow \operatorname{Hom}_{K}\left(M_{j}, M_{j+i}\right), \quad a \rightarrow(m \rightarrow a m)
$$

is injective. 
For any function $f: \mathbb{N} \rightarrow \mathbb{R}$ we can measure the rate of growth of the function by assigning a degree, $\gamma(f)$, to $f$ in the following way.

\section{Definition 3.}

$$
\gamma(f):=\inf \left\{r \in \mathbb{R} \mid f(n) \leq n^{r} \text { for all } n \gg 0\right\}
$$

The first thing that we need to establish is that this degree for the function $n(i)$, just defined, does not depend on the particular choices of filtrations and generating subspaces for $A$ and $M$.

Lemma 4. Let $F$ and $F^{\prime}$ be standard filtrations of an affine algebra $A$, and let $M_{0}$ and $M_{0}^{\prime}$ be finite dimensional generating subspaces of a faithful A-module $M$. Then

$$
\gamma\left(n_{M, F, M_{0}}\right)=\gamma\left(n_{M, F, M_{0}^{\prime}}\right)
$$

Proof. Set $n(i)=n_{M, F, M_{0}}(i)$ and $n^{\prime}(i)=n_{M, F^{\prime}, M_{0}^{\prime}}(i)$. Choose integers $\alpha, \beta$ such that $A_{1} \subseteq A_{\alpha}^{\prime}$ and $A_{1}^{\prime} \subseteq A_{\alpha}$, while $M_{0} \subseteq M_{\beta}^{\prime}$ and $M_{0}^{\prime} \subseteq M_{\beta}$. Then, $M_{i} \subseteq M_{\beta+\alpha i}^{\prime}$ and $M_{i}^{\prime} \subseteq M_{\beta+a i}$, for $i \geq 0$. From this it follows that

$$
n^{\prime}(i) \leq \beta+\alpha n(i \alpha) \text { and } n(i) \leq \beta+\alpha n^{\prime}(i \alpha)
$$

for each $i \geq 0$, and the result follows.

As a consequence of the previous lemma the following definition becomes appropriate.

Definition 5. The left faithful dimension, $\mathrm{f}(M)$, is defined to be

$$
\gamma\left(n_{M, F, M_{0}}\right) \text {, }
$$

for any standard filtration $F$ of $A / \operatorname{ann}(M)$ and for any finite dimensional generating subspace $M_{0}$ of $M$.

The behaviour of this growth on passing to faithful submodules is interesting: it cannot decrease, as the following lemma shows.

Lemma 6. Let $A$ be an affine algebra and let $M, N$ be finitely generated faithful modules with finite dimensional generating subspaces $M_{0}$ and $N_{0}$, respectively. If either (i) $N$ is a submodule of $M$ with $N_{0} \subseteq M_{0}$, or (ii) $N$ is an epimorphic image of $M$ with $N_{0}$ being the image of $M_{0}$ under the epimorphism, then

$$
n_{M, F, M_{0}}(i) \leq n_{N, F, N_{0}}(i)
$$


for $i \geq 0$, and, consequently.

$$
\operatorname{lf}(M) \leq \operatorname{lf}(N)
$$

Proof. This follows easily, for example in case (i) from the fact that if $a \in A$ and $a M_{j}=0$ then $a N_{j}=0$ also, since $N_{j} \subseteq M_{j}$.

Corollary 7. Let $A$ be an affine algebra and let $M, N$ be finitely generated faithful modules with finite dimensional generating subspaces $M_{0}$ and $N_{0}$, respectively. Suppose that $N$ is a subfactor of $M$ (that is, $N \cong X / Y$ for some submodules $Y \subseteq X$ of $M$ ), and suppose that $N_{0} \subseteq M_{0}+Y$. Then

$$
n_{M, F, M_{0}}(i) \leq n_{N, F, N_{0}}(i)
$$

for $i \geq 0$ and, consequently,

$$
\operatorname{lf}(M) \leq \operatorname{lf}(N)
$$

We are now able to establish the following estimate on the relationship between the growth of the algebra $A$ and the growth of the module $M$.

Theorem 8. Let $A$ be an affine algebra and let $M$ be a finitely generated faithful $A$ module. Then

$$
\mathrm{GKdim}(A) \leq \mathrm{GKdim}(M) \times(\operatorname{lf}(M)+\max \{\operatorname{lf}(M), 1\})
$$

Proof. The linear map

$$
A_{i} \rightarrow \operatorname{Hom}_{K}\left(M_{r(i)}, M_{n(i)+i}\right), \quad a \rightarrow(m \rightarrow a m)
$$

is injective, by (2); so that $\operatorname{dim}\left(A_{i}\right) \leq \operatorname{dim}\left(M_{n(i)}\right) \times \operatorname{dim}\left(M_{n(1)+i}\right)$. Using elementary properties of Gelfand-Kirillov dimension, this inequality gives

$$
\begin{aligned}
\operatorname{GKdim}(A) & =\gamma\left(\operatorname{dim}\left(A_{i}\right)\right) \\
& \leq \gamma\left(\operatorname{dim}\left(M_{n(i)}\right)\right)+\gamma\left(\operatorname{dim}\left(M_{n(i)+i}\right)\right) \\
& \leq \gamma\left(\operatorname{dim}\left(M_{i}\right)\right) \gamma(n(i))+\gamma\left(\operatorname{dim}\left(M_{i}\right)\right) \gamma(n(i)+i) \\
& =\operatorname{GKdim}(M) \times(\operatorname{lf}(M)+\max \{\operatorname{lf}(M), 1\})
\end{aligned}
$$

Let $\hat{A}$ be the set of isomorphism classes of simple $A$-modules, and let $\operatorname{Prim}(A)$ be 
the set of primitive ideals of $A$. For a given $J$ in $\operatorname{Prim}(A)$, let $(\widehat{A, J})$ denote the subset of $\hat{A}$ consisting of modules with annihilator equal to $J$.

\section{Definition 9.}

$$
S_{A}:=\inf \left\{\frac{\mathrm{GKdim}(A / J)}{\operatorname{lf}(M)+\max \{\operatorname{lf}(M), 1\}} \mid J \in \operatorname{Prim}(A), M \in(\widehat{A, J})\right\}
$$

Corollary 10. Let $A$ be an affine algebra and let $M$ be a finitely generated $A$-module. Then

$$
\operatorname{GKdim}(M) \geq S_{A},
$$

for any nonzero simple A-module $M$.

The algebra $A$ is said to be left finitely partitive if, given any finitely generated left $A$-module $M$, there is an integer $n>0$ such that for every chain

$$
M \supseteq M_{1} \supseteq M_{2} \supseteq \ldots \supseteq M_{m}
$$

with $\mathrm{GKdim}\left(M_{i} / M_{i+1}\right)=\mathrm{GKdim}(M)$, one has $m \leq n$. Many classes of affine noetherian algebras are known to be finitely partitive. In fact, there are no known examples of such algebras which are not finitely partitive.

Theorem 11. Let $A$ be an affine left finitely partitive algebra such that $\operatorname{GKdim}(A)<\infty$ and that the Gelfand-Kirillov dimension of every finitely generated $A$ module is a natural number. Then,

$$
\operatorname{Kdim}(M) \leq \mathrm{GKdim}(M)-S_{A}
$$

for any finitely generated left A-module $M$.

In particular,

$$
\mathrm{K} \operatorname{dim}(A) \leq \mathrm{GK} \operatorname{dim}(A)-S_{A}
$$

Proof. Let $a \in \mathbb{N}$ and $b \geq 0$ and suppose that $\operatorname{GKdim}(M) \geq a+b$ whenever $M$ is a finitely generated $A$-module such that $\operatorname{Kdim}(M)=a$. Then, $\operatorname{GKdim}(A) \geq \operatorname{Kdim}(A)+b$, and if $N$ is any finitely generated $A$-module with $\operatorname{Kdim}(N) \geq a$ then $G \operatorname{Kdim}(N) \geq \operatorname{Kdim}(N)+b$. Applying this result to the family of finitely generated $A$ modules of Krull dimension zero, where $a=0$, we can set $b=S_{A}$ and the result follows by Corollary 10. 
Holonomic modules

Let $f: \mathbb{N} \rightarrow \mathbb{R}^{1}:=\{r \in \mathbb{R} \mid r \geq 1\}$ be any function. The leading coefficient, $\operatorname{lc}(f)$, of $f$ is the nonzero limit (if it exists)

$$
\operatorname{lc}(f):=\lim _{i \rightarrow \infty} \frac{f(i)}{i^{d}} \quad(\neq 0)
$$

where $d=\gamma(f)$.

Lemma 12. Let $A$ be an affine algebra equipped with the standard finite dimensional filtration $F=\left\{A_{i}\right\}$ and let $M$ be a finitely generated A-module with two finite dimensional generating subspaces $M_{0}$ and $M_{0}^{\prime}$. If $\operatorname{lc}\left(n_{M, F, M_{0}}\right)$ exists then so does $\operatorname{lc}\left(n_{M, F, M_{0}^{\prime}}\right)$, and both numbers are equal. Also, if $\operatorname{lc}\left(\operatorname{dim}\left(A_{i} M_{0}\right)\right)$ exists then so does $\operatorname{lc}\left(\operatorname{dim}\left(A_{i} M_{0}^{\prime}\right)\right)$, and both numbers are equal.

Proof. In the notation of the proof of Lemma 4 , we can put $\alpha=1$; so that $n^{\prime}(i) \leq \beta+n(i)$, and $n(i) \leq \beta+n(i)$, for $i \geq 0$. The first statement follows easily from this observation. The proof of the second statement is standard, see [1, Lemma 3.1].

Thus, since the leading coefficients of $n_{M, F, M_{0}}$ and $\operatorname{dim}\left(A_{i} M_{0}\right)$ do not depend on the choice of the generating subspace $M_{0}$, and since we normally deal with a fixed standard filtration $F$ of $A$, we denote the leading coefficient of the function $n_{M, F, M_{0}}$ by $L(M)=L_{F}(M)$ and the leading coefficient of the function $\operatorname{dim}\left(A_{i} M_{0}\right)$ by $l(M)=l_{F}(M)$.

Definition 13. A finitely generated module $M$ over the affine algebra $A$ is holonomic providing that $G K \operatorname{dim}(M)=S_{A}$. The set of all holonomic modules is denoted by $\operatorname{hol}(A)$.

It is not clear that holonomic modules exist in general. However, if $M$ is a holonomic module then so is any nonzero finitely generated submodule, and any nonzero homomorphic image.

Proposition 14. Let $A$ be an affine algebra with $\mathrm{GKdim}(A)<\infty$ and let $M$ be $a$ simple faithful holonomic $A$-module. If $l(A), l(M)$ and $L(M)$ all exist then

$$
l(A) \leq l(M)^{2}\left(L(M) L^{\prime}(M)\right)^{s_{A}}
$$

where

$$
L^{\prime}(M)= \begin{cases}L(M), & \text { if } \operatorname{lf}(M)>1 \\ L(M)+1, & \text { if } \operatorname{lf}(M)=1 \\ 1, & \text { if } \operatorname{lf}(M)<1\end{cases}
$$


Proof. Let $n(i)=n_{M, F, M_{0}}(A)$ for some finite dimensional generating subspace $M_{0}$ of the module $M$. The embedding (2) of $A_{i}$ into $\operatorname{Hom}_{K}\left(M_{n(i)}, M_{n(1)+i}\right)$, for $i \geq 0$ gives

$$
\operatorname{dim}\left(A_{i}\right) \leq \operatorname{dim}\left(M_{n(i)}\right) \times \operatorname{dim}\left(M_{n(i)+i}\right)
$$

This inequality can be rewritten as

$$
l(A) i^{\mathrm{GKdim}(A)}+\ldots \leq l(M)^{2}\left(L(M) L^{\prime}(M)\right)^{\mathrm{GKdim}(M)} i^{\mathrm{GKdim}(M) \| f(M)+\max \{\mathbb{I f}(M), 1\})}+\ldots
$$

by using the equations

$$
\operatorname{dim}\left(A_{i}\right)=l(A) i^{\mathrm{GKdim}(A)}+\ldots
$$

and

$$
\operatorname{dim}\left(M_{j}\right)=l(M) j^{\mathrm{GKdim}(M)}+\ldots
$$

with $j=n(i)$ and $j=n(i)+i$.

However, $\mathrm{GKdim}(M)=S_{A}$, since $M$ is holonomic, and $\mathrm{GKdim}(A)=\mathrm{GKdim}(M)$ $\{\operatorname{lf}(M)+\max \{\operatorname{lf}(M), 1\}\}$, since $A$ is simple and the infimum in Definition 8 is achieved by $M$.

Hence, the inequality can be written as

$$
l(A) i^{\operatorname{GKdim}(A)}+\ldots \leq l(M)^{2}\left(L(M) L^{\prime}(M)\right)^{S_{A} i^{\mathrm{GKdim}(A)}}+\ldots
$$

and the result follows.

The previous Proposition has the potential to provide a lower bound on $l(M)$. We now look at conditions that are sufficient to establish such a lower bound.

We study affine algebras $A$ that satisfy the following conditions (N), (D) and (H).

(N): There exists a standard finite dimensional filtration $F:=A_{j}$ such that the associated graded algebra $\operatorname{gr}(A):=\oplus A_{\mathrm{i}} / A_{i+1}$ is left noetherian.

(D): $\mathrm{GKdim}(A)<\infty ; l(A / J)=l_{F}(A / J)$ exists, for each $J \in \operatorname{Prim}(A)$, and $\lambda_{A}:=$ $\inf \{l(A / J) \mid J \in \operatorname{Prim}(A)\}>0$. and

(H): For each holonomic $A$-module $M$ both $l(M)=l_{F}(M)$ and $L(M)=L_{F}(M)$ exist,

$$
h_{A}:=\sup \{L(M) \mid M \text { is a simple holonomic module }\}<\infty .
$$

Note that condition (N) guarantees that the algebra $A$ will also be left noetherian.

Condition (H) might seem to be an unreasonable assumption at first sight: there is nothing in the definition of $L(M)$ which suggests a restriction on its size. However, we 
will see, in the section on Differential Operators, that for a well-known class of rings this condition holds.

For an algebra $A$ with these properties, let $c_{A}$ be the positive real number such that

$$
c_{A}^{2}=\frac{\lambda_{A}}{\left(h_{A}\left(h_{A}+1\right)\right)^{s_{A}}} .
$$

Corollary 15. Let $A$ be an algebra with properties $(N),(D)$ and $(H)$. Then

$$
l(M) \geq c_{A}
$$

Proof. This follows immediately from Proposition 14.

Lemma 16. Let $A$ be an algebra with properties $(N),(D)$ and $(H)$. Let $0 \rightarrow N \rightarrow M \rightarrow L \rightarrow 0$ be a short exact sequence of holonomic A-modules. Then $l(M)=l(N)+l(L)$.

Proof. The proof of $[8,8.3 .11]$ shows that one can choose finite dimensional generating subspaces $N_{0}, M_{0}$ and $L_{0}$ of $N, M$ and $L$, respectively, in such a way that, for each $i \geq 0$ there are induced short exact sequences

$$
0 \rightarrow N_{i} \rightarrow M_{i} \rightarrow L_{i} \rightarrow 0
$$

where $N_{i}:=A_{i} N_{0}$, etc. Hence, $\operatorname{dim}\left(M_{i}\right)=\operatorname{dim}\left(N_{i}\right)+\operatorname{dim}\left(L_{i}\right)$, and the result follows.

Theorem 17. Suppose that $A$ is an algebra with properties $(N),(D)$ and $(H)$. Then each holonomic $A$-module has finite length, and this length is less than or equal to $l(M) / c_{A}$.

Proof. Suppose that

$$
M=M_{1} \supset M_{2} \supset \ldots \supset M_{n} \supset M_{n+1}
$$

is a strictly descending sequence of submodules of $M$ such that each subfactor $M_{i} / M_{i+1}$ is simple. Then, by Lemma 16 and Corollary 15 ,

$$
l(M) \geq \sum_{i=1}^{n} l\left(M_{i}\right) / l\left(M_{i+1}\right) \geq n c_{A} .
$$

Hence, $n \leq l(M) / c_{A}$.

A technical point: the important point is to establish a lower bound on the possible 
values of $l(M)$ in order to prove that holonomic modules have finite length and to put a bound on the length, as in Theorem 17. If we re-arrange the inequality in Proposition 14 to read

$$
l(M)^{2} \geq \frac{l(A)}{\left(L(M) L^{\prime}(M)\right)^{S_{A}}},
$$

then we see that it is enough to know that we have an upper bound on the quantity $\frac{n_{M, F, M_{0}(i)}}{i_{0}}$, which is used in the definition of $L(M)$, and a lower bound on $\frac{d \operatorname{dim} A_{M} M_{0}}{i^{d}}$, which is used in the definition of $l(A)$. Thus, if we set $\tilde{L}(M)=\tilde{L}_{F}(M)$ to be the limsup of $\frac{n_{M, F, M_{0}(i)}}{f^{0}}$, or any suitable upper bound, and $\tilde{l}(A)$ to be the liminf of $\frac{\operatorname{dim}\left(\lambda_{1} M_{0}\right)}{A_{0}}$, or any suitable lower bound, together with a similar definition of $\tilde{l}(A / J)$, for each $J \in \operatorname{Prim}(A)$, then we can prove a version of Theorem 17 using these values, providing we replace (D) and $(\mathrm{H})$ by the following conditions.

$\left(\mathrm{D}^{\prime}\right): \operatorname{GKdim}(A)<\infty ; \tilde{l}(A / J)=\tilde{l}_{F}(A / J)$ exists, for each $J \in \operatorname{Prim}(A)$, and $\tilde{\lambda}_{A}:=$ $\inf \{\tilde{l}(A / J) \mid J \in \operatorname{Prim}(A)\}>0$.

$\left(\mathrm{H}^{\prime}\right)$ : For each holonomic $A$-module $M$ both $l(M)=l_{F}(M)$ and $\tilde{L}=\tilde{L}_{F}(M)$ exist, and

$$
\tilde{h}_{A}:=\sup \{\tilde{L}(M) \mid M \text { is a simple holonomic module }\}<\infty .
$$

The point here is that even if the limits exist in a particular example, it may be difficult to get an exact value, but relatively easy to obtain the relevant upper and lower bounds. We record the versions of the previous results under these weaker conditions. The proofs are omitted, since they are merely rewritings of the earlier proofs.

Let $\tilde{c}_{A}$ be defined by

$$
\tilde{c}_{A}^{2}=\frac{\tilde{\lambda}_{A}}{\left(\tilde{h}_{A}\left(\tilde{h}_{A}+1\right)\right)^{S_{A}}} .
$$

Corollary 18. Let $A$ be an algebra with properties $(N),\left(D^{\prime}\right)$ and $\left(H^{\prime}\right)$. Then

$$
l(M) \geq \tilde{c}_{\boldsymbol{A}} .
$$

Lemma 19. Let $A$ be an algebra with properties $(N),\left(D^{\prime}\right)$ and $\left(H^{\prime}\right)$. Let $0 \rightarrow N \rightarrow M \rightarrow L \rightarrow 0$ be a short exact sequence of holonomic A-modules. Then $l(M)=l(N)+l(L)$.

Theorem 20. Suppose that $A$ is an algebra with properties $(N),\left(D^{\prime}\right)$ and $\left(H^{\prime}\right)$. Then each holonomic A-module has finite length, and this length is less than or equal to $l(M) / \tilde{c}_{1}$. 


\section{Comparison with filter dimension}

The left faithful dimension has been introduced mainly to attempt to deal with nonsimple algebras; however, it certainly applies to simple algebras and should be compared in this setting to the left filter dimension introduced by the first author. First, we recall the definition of the left filter dimension.

Let $A$ be a simple affine algebra with a standard filtration $F:=\left\{A_{i}\right\}$. The left return function $\lambda_{F}: \mathbb{N}_{0} \rightarrow \mathbb{N}_{0} \cup\{\infty\}$ of the algebra $A$ is defined as

$$
\lambda_{F}(i):=\min \left\{j \in \mathbb{N}_{0} \cup\{\infty\} \mid 1 \in A a A_{j} \text {, for all } 0 \neq a \in A_{i}\right\}
$$

Definition 21. The degree of $\lambda_{F}$ is called the left filter dimension of $A$, [3], i.e.,

$$
\mathrm{fd}(A):=\gamma\left(\lambda_{F}\right)
$$

The left filter dimension of $A$ does not depend on the choice of $F$.

Lemma 22. Let $A$ be a simple affine algebra and let $M_{0}$ be a finite dimensional generating subspace for an $A$-module $M$. Then

(i) $n_{M, F, M_{0}}(i) \leq \lambda_{F}(i)$, for all $i \geq 0$; hence, $\operatorname{lf}(M) \leq \mathrm{fd}(A)$, and,

$$
\frac{\operatorname{GKdim}(A)}{\mathrm{fd}(A)+\max \{\mathrm{fd}(A), 1\}} \leq S_{A}
$$

(ii) Suppose that

$$
\frac{\operatorname{GKdim}(A)}{\mathrm{fd}(A)+\max \{\mathrm{fd}(A), 1\}}=S_{A}
$$

and that $\operatorname{lc}\left(\lambda_{F}\right)$ exists (respectively, $\left.\tilde{\operatorname{lc}}\left(\lambda_{F}\right):=\lim \sup \frac{\lambda_{F}(i)}{i_{1}^{5}}\right)$ then, for any holonomic $A-$ module $M$,

$$
L_{F}(M) \leq \operatorname{lc}\left(\lambda_{F}\right) \quad\left(\text { respectively } \tilde{L}_{F}(M) \leq \tilde{\operatorname{lc}}\left(\lambda_{F}\right)\right)
$$

Hence,

$$
h_{A} \leq \operatorname{lc}\left(\lambda_{F}\right) \quad\left(\text { respectively } \tilde{h}_{A} \leq \overline{\operatorname{lc}}\left(\lambda_{F}\right)\right)
$$

Proof. (i) This follows the argument of [2, Theorem 1]. From the fact that $1 \in A a A_{\lambda(1)}$, setting $\lambda=\lambda_{F}$, we obtain 


$$
M_{0}=1 M_{0} \subseteq A a A_{\lambda(i)} M_{0}=A a M_{\lambda(i)},
$$

and, hence, the linear map

$$
A_{i} \rightarrow \operatorname{Hom}_{K}\left(M_{\lambda(i)}, M_{\lambda(i)+i}\right), \quad a \rightarrow(m \rightarrow a m),
$$

is injective. Thus, $n_{M, F, M_{0}}(i) \leq \lambda_{F}(i)$, for all $i \geq 0$, as required.

(ii) This is evident.

Remark 23. Part (ii) of the previous lemma shows that the condition (H) is not as restrictive as it might seem.

\section{Differential operators}

Let $B$ be a commutative regular integral domain of Krull dimension $n$, affine over a field $K$ of characteristic zero. Let $\mathcal{D}(B)$ be its ring of differential operators. It is wellknown that $\mathcal{D}(B)$ is a simple affine noetherian algebra, [8, Chapter 15]. Also,

$$
\operatorname{Kdim}(\mathcal{D}(B))=\frac{\operatorname{GKdim}(\mathcal{D}(B))}{2}=n
$$

and

$$
\frac{G K \operatorname{dim}(\mathcal{D}(B))}{2} \leq \mathrm{GKdim}(M)
$$

for any nonzero finitely generated $\mathcal{D}(B)$-module $M$. If there is equality in this inequality then the module $M$ is holonomic.

In this setting, $\mathcal{D}(B)$ has well-behaved growth properties. It is pointed out in [8, 15.1.21] that $\mathcal{D}(B)$ is a somewhat commutative algebra, and it then follows from [9] that each finitely generated module has a rational Hilbert Series, and hence integer Gelfand-Kirillov dimension and a well-defined leading coefficient. Further, the first author has shown in [3] that the left filter dimension of $\mathcal{D}(B)$ is one. This follows from the next lemma, which gives more specific information.

Let $\left\{B_{i}\right\}$ and $F:=\left\{\mathcal{D}(B)_{i}\right\}$ be standard finite dimensional filtrations on $B$ and $\mathcal{D}(B)$, respectively, such that $B_{i} \subseteq \mathcal{D}(B)_{i}$, for all $i \geq 0$. The enveloping algebra $\mathcal{D}(B)^{e}:=$ $\mathcal{D}(B) \otimes \mathcal{D}(B)^{\circ}$, where $\mathcal{D}(B)^{\circ}$ is the opposite algebra, can be equipped with a standard finite dimensional filtration $\left\{\mathcal{D}(B)_{i}^{e}\right\}$, which is the tensor product of the filtrations $F$ and $F^{o}$. Note that the algebra $\mathcal{D}(B)$ is a filtered left $\mathcal{D}(B)^{e}$-module.

Lemma $24[2,2.1]$. There exist natural numbers $a$ and $b$ such that for any $d \in \mathcal{D}(B)_{i}$ there exists $w \in \mathcal{D}(B)_{a i+b}^{e}$ satisfying $w d=1$.

Note that this result establishes that $\mathcal{D}(B)$ is a simple algebra in a strong sense: the 
necessary elements to show that any element $d$ generates $\mathcal{D}(B)$ as a two sided ideal can be found a linear distance up in the standard filtration. This lemma is also useful in the following result which demonstrates that $\tilde{h}_{D(B)}$ is finite in this class of algebras, and also demonstrates that our new notion of holonomic coincides with the old notion in this important class of rings.

Corollary 25. Let $M$ be a holonomic $\mathcal{D}(B)$-module. Then $\operatorname{lf}(M)=1$ and $L(M) \leq a$, so that

$$
\tilde{h}_{\mathcal{D}(B)} \leq a<\infty
$$

Proof. It follows from Theorem 8 and (5) that $\operatorname{lf}(M) \geq 1$ and from the previous lemma that $\operatorname{lf}(M) \leq 1$. Also, there is an embedding

$$
\mathcal{D}(B)_{i} \hookrightarrow \operatorname{Hom}\left(M_{a i+b}, M_{a i+b+i}\right), \quad u \rightarrow(m \rightarrow u m),
$$

so that $\tilde{L}(M) \leq a$.

The above corollary shows: firstly, each classical holonomic module for the ring $\mathcal{D}(B)$ is holonomic in the sense of Definition 13, and vice-versa; secondly, that requiring the finiteness of $h_{A}\left(\right.$ or $\tilde{h}_{A}$ ) is a reasonable restriction, in that it certainly holds for this important class of rings.

\section{Schurian modules}

Let $K$ be an algebraically closed field and let $A$ be an affine $K$-algebra. An $A$-module $M$ is called schurian if $\operatorname{End}_{A}(M)=K$. An algebra $A$ is said to be schurian if each simple $A$-module is schurian.

The class of schurian algebras is a wide class of algebras containing many interesting and important rings. For example, if the field $K$ is uncountable and algebraically closed then all affine algebras are schurian. (In fact, they satisfy the Nullstellensatz, a stronger requirement, see [8, Chapter 9].) In addition, for any algebraically closed field $K$, any constructible algebra is schurian; again, see [8, Chapter 9].

Let $A$ be an affine algebra with standard finite dimensional filtration $F=\left\{A_{i}\right\}$ and let $M$ be a faithful schurian simple module with standard finite dimensional filtration $\left\{M_{i}=A_{i} M_{0}\right\}$, where $M_{0}$ is a finite dimensional generating subspace of $M$. The map

$$
A \rightarrow \operatorname{Hom}_{K}(M, M), \quad a \rightarrow(m \rightarrow a m),
$$

is injective, since the module $M$ is faithful. We identify $A$ with its image under this injection, and note that $A$ acts as a dense ring of $K$-linear transformations, since $M$ is a faithful schurian simple $A$-module. This terminology will be used throughout this section. 
Consider the following function.

\section{Definition 26.}

$$
\mu(i):=\mu_{M, F, M_{0}}(i)=\min \left\{j \mid \operatorname{Hom}_{K}\left(M_{i}, M_{i}\right) \subseteq A_{j}\right\},
$$

where the inclusion above means that for any $K$-linear map $\phi: M_{i} \rightarrow M_{i}$ there exists an element $a \in A_{j}$ such that

$$
\phi(m)=a m,
$$

for all $m \in M_{i}$.

In this notation,

$$
\operatorname{Hom}_{K}\left(M_{i}, M_{i}\right) \subseteq A_{\mu(0)}
$$

and so

$$
\left(\operatorname{dim}\left(M_{i}\right)\right)^{2} \leq \operatorname{dim}\left(A_{\mu(i)}\right),
$$

As usual, we need to check that the rate of growth of this function is independent of the standard filtrations involved.

Lemma 27. Let $F$ and $F^{\prime}$ be standard filtrations of an affine algebra $A$, and let $M_{0}$ and $M_{0}^{\prime}$ be finite dimensional generating subspaces of a faithful simple schurian A-module M. Then

$$
\gamma\left(\mu_{M, F, M_{0}}\right)=\gamma\left(\mu_{M, F^{\prime}, M_{0}^{\prime}}\right)
$$

Proof. Set $\mu=\mu_{M, F, M_{0}}$ and $\mu^{\prime}=\mu_{M, F, M_{0}^{\prime}}$. Let $\alpha, \beta$ be as in the proof of Lemma 4 . Then $M_{i} \subseteq M_{\beta+\alpha t}^{\prime}$ and $M_{i}^{\prime} \subseteq M_{\beta+\alpha i}$, and similarly, $A_{i} \subseteq A_{\alpha i}^{\prime}$ and $A_{i}^{\prime} \subseteq A_{a i}$, for all $i \geq 0$.

Thus,

$$
\operatorname{Hom}_{K}\left(M_{i}, M_{i}\right) \subseteq \operatorname{Hom}_{K}\left(M_{\beta+\alpha i}^{\prime}, M_{\beta+\alpha i}^{\prime}\right) \subseteq A_{\mu(\beta+\alpha)}^{\prime} \subseteq A_{\beta+\alpha \mu^{\prime}(\beta+\alpha i)}
$$

Hence,

$$
\mu(i) \leq \beta+\alpha \mu^{\prime}(\beta+\alpha i)
$$

for $i \geq 0$; and so $\gamma(\mu) \leq \gamma\left(\mu^{\prime}\right)$. The opposite inequality follows by a symmetrical argument.

Definition 28. The Schur dimension, $\operatorname{sd}(M)$, of a module $M$ is given by 


$$
\operatorname{sd}(M):=\gamma\left(\mu_{M, F, M_{0}}\right)
$$

Theorem 29. Let $A$ be an affine algebra and let $M$ be a faithful simple schurian $A$ module. Then

$$
\operatorname{GKdim}(M) \leq \frac{\mathrm{GKdim}(A)}{2} \operatorname{sd}(M)
$$

Proof. Using (8), we have

$$
\begin{aligned}
2 \mathrm{GKdim}(M) & =\gamma\left(\left(\operatorname{dim}\left(M_{i}\right)\right)^{2}\right) \leq \gamma\left(\operatorname{dim}\left(A_{\mu(i)}\right)\right) \\
& \leq \gamma\left(\operatorname{dim}\left(A_{i}\right)\right) \gamma(\mu)=\mathrm{GKdim}(A) \operatorname{sd}(\mu) .
\end{aligned}
$$

Corollary 30. Let $A$ be an affine algebra and let $M$ be a faithful simple schurian $A$-module, and suppose that $M$ is not a finite dimensional $A$-module, so that $\operatorname{GKdim}(M)>0$. Then

$$
2 \leq \operatorname{sd}(M)\{\operatorname{lf}(M)+\max \{\operatorname{lf}(M), 1\}\}
$$

Proof. The claim follows from the previous theorem and Theorem 8 .

Now, fix a filtration $F=\left\{A_{i}\right\}$.

Lemma 31. Let $A$ be an affine algebra and let $M$ be a faithful simple schurian $A$ module. Let $M_{0}$ and $M_{0}^{\prime}$ be two finite dimensional generating subspaces of the module $M$. If the leading coefficient $\operatorname{lc}\left(\mu_{M, F, M_{0}}\right)$ exists then so does $\operatorname{lc}\left(\mu_{M, F, M_{0}^{\prime}}\right)$, and the two numbers are equal.

Proof. In the notation of Lemma 27, we can put $\alpha=1$; and so $\mu(i) \leq \beta+\mu^{\prime}(i+\beta)$ and $\mu^{\prime}(i) \leq \beta+\mu(i+\beta)$, for $i \geq 0$. The result then follows easily.

Definition 32. Set

$$
\mathcal{L}(\mu)=\mathcal{L}\left(\mu^{\prime}\right):=\operatorname{lc}\left(\mu_{M, F, M_{0}}\right)
$$

Theorem 33. Let $A$ be an affine algebra with $\operatorname{GKdim}(A)<\infty$ and let $M$ be a faithful simple schurian $A$-module. Suppose that each of $l(A), l(M)$ and $\mathcal{L}(M)$ exists and that $\operatorname{GKdim}(M)=\frac{\mathrm{GKdim}(1)}{2} \mathrm{sd}(M)$. Then

$$
\frac{l(M)^{2}}{\mathcal{L}(M)^{\mathrm{GKdim}(A)}} \leq l(A)
$$


Proof. Let $\mu(i)=\mu_{M, F, M_{0}}(i)$, for some finite dimensional generating subspace $M_{0}$ of $M$. The inequality (8) can be rewritten as

$$
l(M)^{2} i^{2 \mathrm{GKdim}(M)}+\ldots \leq l(A) \mathcal{L}(M)^{\mathrm{GKdim}(A)} i^{\mathrm{GKdim}(A) \operatorname{sd}(M)}+\ldots
$$

Comparing leading coefficients, using the assumption that $\operatorname{GKdim}(M)=\frac{\operatorname{GKdim}(1)}{2} \operatorname{sd}(M)$, we obtain

$$
l(M)^{2} \leq l(A) \mathcal{L}(M)^{\mathrm{GKdim}(A)}
$$

Let $B$ be a commutative regular integral domain of Krull dimension $n$, affine over an algebraically closed field $K$ of characteristic zero. Let $\mathcal{D}(B)$ be its ring of differential operators. Then $\mathcal{D}(B)$ is a schurian algebra.

Corollary 34. If $M$ is a simple $\mathcal{D}(B)$-module, then $\operatorname{sd}(M) \geq 1$. If $\operatorname{sd}(M)=1$, then $M$ is holonomic.

Proof. The statement follows from the inequalities

$$
\frac{\operatorname{GKdim}(\mathcal{D}(B))}{2} \leq \mathrm{GKdim}(M) \leq \frac{\mathrm{GKdim}(\mathcal{D}(B))}{2} \mathrm{sd}(M)
$$

Remark 35. In contrast, the famous simple nonholonomic modules for the Weyl algebra $A_{n}$, for $n \geq 2$, discovered by Stafford, [11] or [5, Theorem 8.7, Proposition 8.8], have $\operatorname{sd}(M)>1$.

\section{An example}

Let $K$ be an algebraically closed field and let $D:=K\left[H_{1}^{ \pm 1}, \ldots, H_{n}^{ \pm 1}\right]$ be the commutative Laurent polynomial ring in $n$ indeterminates. Let $\lambda_{1}, \ldots, \lambda_{n} \in K$ be such that the multiplicative subgroup of $K$ generated by $\lambda_{1}, \ldots, \lambda_{n}$ is free abelian of rank $n$. Set $A=A_{n}:=D\left[X^{ \pm 1} ; \sigma\right]$, the skew-Laurent polynomial ring, where $\sigma\left(H_{i}\right)=\lambda_{i}^{-1} H_{i}$, so that $X H_{i}=\lambda_{i}^{-1} H_{i} X$. Alternatively, we can present $A$ as $A=K\left[X^{ \pm 1}\right]$ $\left[H_{1}^{ \pm 1}, \ldots, H_{n}^{ \pm 1} ; \sigma_{1}, \ldots, \sigma_{n}\right]$ where $\sigma_{i}(X)=\lambda_{i} X$.

The algebra $A=\oplus_{i \in \mathbf{Z}} A^{i}$ is a central simple $\mathbb{Z}$-graded algebra, where $A^{i}:=D X^{i}$, for $i \in \mathbb{Z}$.

The algebras $A$ and $D$ have standard filtrations

$$
A=\bigcup_{i \geq 0} A_{i}, \quad A_{i}:=A_{1}^{i}, \quad A_{1}:=\sum_{i=1}^{n} K H_{i}+\sum_{i=1}^{n} K H_{i}^{-1}+K X+K X^{-1}
$$

and 


$$
D=\bigcup_{i \geq 0} D_{i}, \quad D_{i}:=D_{1}^{i}, \quad D_{1}:=\sum_{i=1}^{n} K H_{i}+\sum_{i=1}^{n} K H_{i}^{-1} .
$$

Clearly,

$$
A_{i}=\oplus\left\{K H^{\alpha} X^{j}|| \alpha|+| j \mid \leq i\right\} \quad \text { and } \quad D_{i}=\oplus\left\{K H^{\alpha}|| \alpha \mid \leq i\right\}
$$

where $H^{\alpha}:=H_{1}^{\alpha_{1}} \ldots H_{n}^{\alpha_{n}},|\alpha|=\left|\alpha_{1}\right|+\ldots+\left|\alpha_{n}\right|$, and $D_{i}=D \cap A_{i}$ for $i \geq 0$. It can easily be checked that $\operatorname{dim}\left(D_{i}\right)$ is a polynomial with leading term

$$
\operatorname{dim}\left(D_{i}\right)=\frac{2^{n}}{n !} i^{n}+\ldots
$$

The map

$$
\operatorname{Maxspec}(D) \rightarrow\left(K^{*}\right)^{n}, \quad \mathcal{M}_{\mu}:=\left\langle H_{1}-\mu_{1}, \ldots, H_{n}-\mu_{n}\right\rangle \rightarrow \mu=\left(\mu_{1}, \ldots, \mu_{n}\right),
$$

is a bijection.

For each $\mu$, consider the simple $D$-module $V \equiv V_{\mu}:=D / \mathcal{M}_{\mu} \cong K$. The induced module

$$
A(V):=A \otimes_{D} V=\bigoplus_{i \in \mathbf{Z}} K e_{i}, \quad e_{i}:=X^{i} \otimes \overline{1}, \quad \overline{1}=1+\mathcal{M}_{\mu} \in V
$$

is a $\mathbb{Z}$-graded module:

$$
A(V)=\bigoplus_{i \in \mathbf{Z}} A(V)^{i}, \quad A(V)^{i}:=K e_{i}
$$

The action of elements of $A$ on this induced module is as follows: $X e_{i}=e_{i+1}$ and $H^{\alpha} e_{i}=\mu^{\alpha} \lambda^{i \alpha} e_{i}$, for all $\alpha=\left(\alpha_{1}, \ldots, \alpha_{n}\right) \in \mathbb{Z}^{n}$, where $H^{\alpha}=\prod H_{j}^{\alpha_{j}}, \mu^{\alpha}=\prod \mu_{j}^{\alpha_{j}}, \lambda^{i \alpha}=\prod \lambda_{j}^{i \alpha_{j}}$.

Each graded component $A(V)^{i}$ is a simple $D$-module that is isomorphic to $D / \sigma^{i}\left(\mathcal{M}_{\mu}\right)$. The $A$-module $A(V)$ is equipped with the standard filtration

$$
A(V)=\bigcup_{i=0}^{\infty} A(V)_{i}
$$

where

$$
A(V)_{i}=A_{i} \cdot A(V)_{0}=\bigoplus_{-i \leq j \leq i} K e_{j}
$$

It follows that $\mathrm{GKdim}(A(V))=1$, since $\operatorname{dim}\left(A(V)_{i}\right)=2 i+1$.

The $D$-modules $A(V)^{i}$, for $i \in \mathbb{Z}$, are pairwise non-isomorphic, by the choice of $\left\{\lambda_{i}\right\}$. 
Hence, the $A$-module $A(V)$ is simple, since $X$ acts bijectively on $A(V)$, and the $H^{\alpha}$ act on $e_{i}$ by distinct scalars.

Lemma 36. For every simple $D$-module $V_{\mu}$, the $A$-module $A\left(V_{\mu}\right)$ is a simple $A$-module of Gelfand-Kirillov dimension one. Two such $A$-modules $A\left(V_{\mu}\right)$ and $A\left(V_{v}\right)$ are isomorphic if and only if $\mathcal{M}_{v}=\sigma^{i}\left(\mathcal{M}_{\mu}\right)$, for some $i \in \mathbb{Z} ;$ that is, $v_{j}=\lambda_{j}^{i} \mu_{j}$, for all $j$ and for some $i \in \mathbb{Z}$.

Lemma 37. Let $M=A(V)$ be as above. The natural map

$$
A_{i} \rightarrow \operatorname{Hom}_{K}\left(M_{j}, M_{j+i}\right), \quad a \rightarrow(\hat{a}: m \rightarrow a m)
$$

is injective if and only if the natural map

$$
D_{i} \rightarrow \operatorname{Hom}_{K}\left(M_{j}, M_{j}\right), \quad d \rightarrow(\hat{d}: m \rightarrow d m)
$$

is injective.

Proof. $\quad \Rightarrow$ The second map is the restriction of the first map to $D_{i}$, since the $A$ module $M$ is $\mathbb{Z}$-graded and $D_{i} \subseteq A_{0}$, so that $D_{i} M_{j} \subseteq M_{j}$.

$(\Leftarrow)$ Let $a=a_{0}+X a_{1}+\ldots+X^{k} a_{k}$, with $a_{i} \in D$, belong to the kernel of the first map. Then $a e_{i}=0$, for all $i=0, \ldots, \pm j$, or, equivalently, $X^{m} a_{m} e_{i}=0$, for all possible $m, i$ (since $M$ is $\mathbb{Z}$-graded and $e_{i} \in M^{i}$ ). Observe that $\hat{X}$ is a bijection, hence, $\hat{a}_{m}=0$, for all $m$; that is, $\hat{a}=0$.

Proposition 38. Let $\mu=\left(\mu_{1}, \ldots, \mu_{n}\right) \in\left(K^{*}\right)^{n}$ and let $V$ be the D-module $D /\left\langle H_{1}-\mu_{1}, \ldots, H_{n}-\mu_{n}\right\rangle$. Then the left faithful dimension of the A-module $A(V)=A \otimes_{D} V$ is $n$.

Proof. Set $M=A(V)$ and keep the notation introduced above. Let $n(i)$ be the function $n_{M, F, M_{0}}(i)$ defined in (1), where $F=\left\{A_{i}\right\}$ and $M_{0}=K e_{0}$. The module $M$ is $\mathbb{Z}$ graded and $D \subseteq A_{0}$, so, by (2) we have the inclusion

$$
D_{i} \hookrightarrow \operatorname{Hom}_{K}\left(M_{n(i)}, M_{n(i)}\right), \quad d \rightarrow(\hat{d}: d \rightarrow d m) .
$$

Using the basis $\left\{e_{i} \mid i=0, \pm 1, \ldots, \pm n(i)\right\}$ of the $K$-vector space $M_{n(i)}$, we may identify $\operatorname{Hom}_{K}\left(M_{m(i)}, M_{n(i)}\right)$ with the matrix ring of $\operatorname{dim}\left(M_{n(i)}\right) \times \operatorname{dim}\left(M_{m(i)}\right)$ matrices over $K$. Under this identification, every $\hat{d}$ is identified with a diagonal matrix, so, in fact, we have an inclusion

$$
D_{i} \rightarrow \operatorname{Diag}_{\operatorname{dim}\left(M_{\text {a(t) }}\right)}(K)
$$

of $D_{i}$ into a vector space of dimension $\operatorname{dim}\left(M_{n(i)}\right)=2 n(i)+1$. Hence, $n=\gamma\left(\operatorname{dim}\left(D_{i}\right)\right) \leq$ $\gamma(n(i))=\operatorname{lf}(M)$, so that $n \leq \operatorname{lf}(M)$.

Next, we prove the opposite inequality. The idea is to show that the map 


$$
D_{i} \rightarrow \operatorname{Hom}_{K}\left(M_{d(i)-1}^{+}, M_{d(i)-1}^{+}\right), \quad d \rightarrow \hat{d}
$$

is injective, where $d(i)=\operatorname{dim}\left(D_{i}\right)$ and $M_{d(i)-1}^{+}=\oplus_{0 \leq j \leq d(i)-1} K e_{j}$ is a subspace of $M_{d(i)}$. Suppose for the moment that we have already achieved this, so that the above map is injective. Then, by the previous lemma, $n(i) \leq d(i)$, so that

$$
\operatorname{lf}(M)=\gamma(n(i)) \leq \gamma\left(\operatorname{dim}\left(D_{i}\right)\right)=n,
$$

and so $\operatorname{lf}(M)=n$.

Fix the basis $\left\{e_{j} \mid j=0, \ldots, d(i)-1\right\}$ of the space $M_{d(i)-1}^{+}$. By repeating the argument above, the algebra homomorphism

$$
D_{i} \rightarrow \operatorname{Hom}_{K}\left(M_{d(\mathfrak{i})-1}^{+}, M_{d(\mathfrak{l})-1}^{+}\right), \quad d \rightarrow \hat{d}
$$

can be viewed as the algebra homomorphism

$$
\psi: D_{i} \rightarrow \operatorname{Diag}_{d(i)}(K) \cong K^{d(i)}, \quad H^{\alpha} \rightarrow \psi\left(H^{\alpha}\right)=\mu^{\alpha}\left(1, \lambda^{\alpha}, \lambda^{2 \alpha}, \ldots, \lambda^{(d(i)-1) \alpha}\right)
$$

The basis $\left\{H^{\alpha}\right\}$ of $D_{i}$ can be ordered lexicographically, and then the $d(i) \times d(i)$ matrix $[\psi]$ of the map $\psi$ has rows $\psi\left(H^{\alpha}\right)$ and so is of the form

$$
\left|\begin{array}{cccccc}
\mu^{\alpha_{0}} & \mu^{\alpha_{0}} & \mu^{\alpha_{0}} & \ldots & \mu^{\alpha_{0}} & \mu^{\alpha_{0}} \\
\mu^{\alpha_{1}} & \mu^{\alpha_{1}} \lambda^{\alpha_{1}} & \mu^{\alpha_{1}} \lambda^{2 \alpha_{1}} & \ldots & \mu^{\alpha_{1}} \lambda^{(d(\hat{n})-2) \alpha_{1}} & \mu^{\alpha_{1}} \lambda^{(d(\hat{)})-1) \alpha_{1}} \\
\mu^{\alpha_{2}} & \mu^{\alpha_{2}} \lambda^{\alpha_{2}} & \mu^{\alpha_{2}} \lambda^{2 \alpha_{2}} & \ldots & \mu^{\alpha_{2}} \lambda^{(d(i)-2) \alpha_{2}} & \mu^{\alpha_{2}} \lambda^{(d(\hat{)})-1) \alpha_{2}} \\
\vdots & \vdots & \vdots & \vdots & \vdots & \vdots \\
\vdots & \vdots & \vdots & \vdots & \vdots & \vdots \\
\mu^{\alpha_{n-1}} & \mu^{\alpha_{n-1}} \lambda^{\alpha_{n-1}} & \mu^{\alpha_{n-1}} \lambda^{2 \alpha_{n-1}} & \ldots & \mu^{\alpha_{n-1}} \lambda^{(d(\hat{)})-2) \alpha_{n-1}} & \mu^{\alpha_{n-1}} \lambda^{(d(\hat{)})-1) \alpha_{n-1}}
\end{array}\right|
$$

where $n=d(i)$. This is almost a Van der Monde determinant, and so is easy to evaluate. We have,

$$
\operatorname{Det}[\psi]=\prod \mu^{\alpha} \cdot \prod_{\alpha>\beta}\left(\lambda^{\alpha}-\lambda^{\beta}\right)
$$

where $\lambda^{0}=1$.

Thus $\operatorname{Det}[\psi] \neq 0$, since $\lambda^{\alpha} \neq \lambda^{\beta}$, for $\alpha \neq \beta$. Hence the map $\psi$ is an algebra monomorphism.

The algebra $A$ can also be described as the skew Laurent extension $A=K\left[X^{ \pm 1}\right]\left[H_{1}^{ \pm 1}, \ldots, H_{n}^{ \pm 1} ; \sigma_{1}, \ldots, \sigma_{n}\right]$ where $\sigma_{i}(X)=\lambda_{i} X$. Set $R:=K\left[X^{ \pm 1}\right]$. The algebra $A$ is a $\mathbb{Z}^{n}$-graded algebra via 


$$
A=\bigoplus_{\alpha \in Z^{n}} A_{x}, \quad A_{x}=R H^{\alpha}
$$

For $\mu \in K^{*}$, consider the induced $A$-module formed from the simple $R$-module $U_{\mu}:=R /(X-\mu) \cong K$. So

$$
A\left(U_{\mu}\right):=A \otimes_{R} U_{\mu}=\bigoplus_{a \in \mathbb{Z}^{n}} K e_{a}, \quad e_{\alpha}=H^{\alpha} \otimes \overline{1}, \quad \overline{1}=1+\langle X-\mu\rangle
$$

The module $A\left(U_{\mu}\right)$ is $\mathbb{Z}^{n}$-graded by $A\left(U_{\mu}\right)_{\alpha}:=K e_{\alpha}$, for $\alpha \in \mathbb{Z}^{n}$. The action of elements of $A$ on $A\left(U_{\mu}\right)$ is defined by $H^{\beta} e_{\alpha}=e_{\alpha+\beta}$ and $X e_{\alpha}=\mu \lambda^{-x} e_{\alpha}$, for $\alpha, \beta \in \mathbb{Z}^{n}$. As a $D$-module, $A\left(U_{\mu}\right)$ is free of rank 1 . The standard filtration on $A\left(U_{\mu}\right)=\cup_{i=0}^{\infty} A\left(U_{\mu}\right)_{i}$, where $A\left(U_{\mu}\right)_{i}=A_{i} e_{0}=D_{i} e_{0}$, "coincides" with the standard filtration of the algebra $D$, so that $\operatorname{dim} A\left(U_{\mu}\right)_{i}=\operatorname{dim} D_{i}=\frac{2^{n}}{n !} i^{n}+\ldots$, and so $\mathrm{GKdim} A\left(U_{\mu}\right)=n$.

Repeating the arguments as in the previous case, we establish the following lemma.

Lemma 39. The A-module $A\left(U_{\mu}\right)$ is simple of Gelfand-Kirillov dimension $n$, for each $\mu \in K^{*}$. Two such modules $A\left(U_{\mu}\right)$ and $A\left(U_{v}\right)$ are isomorphic if and only if $v=\lambda^{\alpha} \mu$, for some $\alpha \in \mathbb{Z}^{n}$.

Proposition 40. Let $\mu \in K^{*}$, and set $U_{\mu}=R /(X-\mu\rangle$. The left faithful dimension of $A\left(U_{\mu}\right)=A \otimes_{R} U_{\mu}$ is $\frac{1}{n}$.

Proof. Let $d_{i}=\operatorname{dim}\left(A\left(U_{\mu}\right)_{i}\right)=\operatorname{dim}\left(D_{i}\right)=\frac{2^{n}}{n^{n}} i^{n}+\ldots$. We will show that the following map is an inclusion:

$$
A_{d_{i}-1} \rightarrow \operatorname{Hom}_{k}\left(A\left(U_{\mu}\right)_{i}, A\left(U_{\mu}\right)_{i+d_{i}-1}\right), \quad a \rightarrow \hat{a} .
$$

Once this has been demonstrated, it follows that

$$
\operatorname{lf}\left(A\left(U_{\mu}\right)\right) \leq \frac{1}{n}
$$

On the other hand, by (3),

$$
1+\frac{1}{n}=\frac{n+1}{n}=\frac{\mathrm{GKdim}(A)}{\mathrm{GKdim}\left(A\left(U_{\mu}\right)\right)} \leq \operatorname{lf}\left(A\left(U_{\mu}\right)\right)+\max \left\{\operatorname{lf}\left(A\left(U_{\mu}\right)\right), 1\right\}=\operatorname{lf}\left(A\left(U_{\mu}\right)\right)+1,
$$

so If $\left(A\left(U_{\mu}\right)\right) \geq \frac{1}{n}$. Therefore, if $\left(A\left(U_{\mu}\right)\right)=\frac{1}{n}$.

Suppose that $a=\sum f_{\alpha} H^{\alpha}$, where $f_{a}^{n} \in K\left[X^{ \pm 1}\right]$ belongs to the kernel of the map above; that is, $\hat{a}=0$. The modules $A\left(U_{\mu}\right)$ is $\mathbb{Z}^{n}$-graded and isomorphic to $D$ as a $D$-module, so $\hat{a}=0$ if and only if each $\hat{f}_{a}=0$. This implies that the polynomial $g_{i}=\prod_{|x| \leq i}\left(X-\mu \lambda^{-\alpha}\right)$ divides each $f_{\alpha}$. However, $\operatorname{deg}\left(g_{i}\right)=d_{i}$, while $f_{\alpha} \in A_{d_{i}-1}$, a contradiction. 


\section{REFERENCES}

1. V. BAvULA, Filter dimension of algebras and modules, a simplicity criterion of generalized Weyl algebras, Comm. Algebra 24 (1996), 1971-1992.

2. V. Bavula, Krull, Gelfand-Kirillov and filter dimensions of simple affine algebras, $J$. Algebra 206 (1998), 33-39.

3. V. Bavula, Krull and Gelfand-Kirillov dimensions of simple almost commutative algebras.

4. I. N. Bernstein, The analytic continuation of generalized functions with respect to a parameter, Funct. Anal. Appl. 6 (1972), 273-285.

5. G. R. KRAUSE and T. H. Lenagan, Growth of algebras and Gelfand-Kirillov dimension (Pitman, 1985).

6. A. JosEPh, Applications de la Thèorie des anneaux algèbres enveloppantes, Cours de troisième cycle. Univ. de Paris VI, mimeographed notes, 1981.

7. J. C. MCCONNELL, Representations of solvable Lie algebras V: On the Gelfand-Kirillov dimension of simple modules, J. Algebra 76 (1982), 489-493.

8. J. C. MCConnell and J. C. Robson, Noncommutative noetherian rings (Wiley, 1987).

9. J. C. MCConNell AND J. C. RoBson, Gelfand-Kirillov dimension, Hilbert-Samuel polynomials and rings of differential operators, Perspectives in ring theory (Antwerp, 1987), 233238, NATO Adv. Sci. Inst. Ser. C: Math. Phys. Sci., 233, Kluwer Acad. Publ., Dordrecht, 1988.

10. S. P. SMrTH, Krull dimension of the enveloping algebra of $s l(2, \mathbb{C}), J$. Algebra 71 (1981), 89-94.

11. J. T. STAFford, Non-holonomic modules over Weyl algebras and enveloping algebras, Invent. Math. 79 (1985), 619-638.

\author{
DePartMent OF Mathematics \\ KIEV UNIVERSITY \\ VladimirsKaya STR, 64 \\ KIEV 252617 \\ UKRAINE \\ E-mail address: sveta@,kinr.kiev.ua
}

\author{
Department of Mathematics \\ UNIVERSITY OF EDINBURGH \\ James Clerk MaXwell Building \\ KING'S BUILDINGS \\ MAYFIELD ROAD \\ EDINBURGH EH9 3JZ \\ E-mail address: tom@maths.ed.ac.uk
}

